\title{
Evidence of Instanton Effects in Hadrons from the Study of Low Eigenfunctions of the Dirac Operator
}

\author{
T.L. Ivanenko and J.W. Negele ${ }^{\mathrm{a} *}$ \\ ${ }^{a}$ Center for Theoretical Physics \\ Laboratory for Nuclear Science and Department of Physics \\ Massachusetts Institute of Technology, Cambridge MA 02139
}

MIT-CTP\#2677 hep-lat/9709130

To elucidate the role in hadron structure of quark zero modes associated with instantons, the lowest eigenfunctions of the Dirac operator have been calculated in quenched QCD, full QCD, and full QCD with cooling. Eigenmodes associated with a single instanton and an instanton-anti-instanton pair were studied to understand the role of lattice artifacts for Wilson Fermions. By truncating the spectral representation of the quark propagator, we show that the rho and pion contributions to the vector and pseudoscalar correlation functions are dominated by the zero-mode contributions.

\section{Introduction and Motivation}

This work is part of an ongoing effort to use the lattice to obtain insight into the structure of light hadrons by finding the paths that dominate the QCD path integral[i]. The picture that emerges is that of the instanton liquid developed by Shuryak 2] and by Dyakanov and Petrov [3]. In this picture, light quarks propagate by hopping between zero modes, and the resulting 't Hooft interaction [4] accounts for many phenomena, ranging from the channel dependence of vacuum hadron current correlation functions [5] to the so-called "spin crisis" in which helicity is transferred from valence quarks to gluons and $\bar{q} q$ excitations and the $\bar{d} d$ to $\bar{u} u$ ratio in the proton. Previous calculations show that removing noninstanton components of the gluon configurations by cooling[6] reproduces the essential features of the instanton model and observables calculated with all gluons are similar to those obtained using only instantons that survive cooling[7].

The chief limitation of these cooling studies is that although the loss of a single instanton by cooling can be avoided by the use of an improved action [8], the annihilation of instantonanti-instanton pairs is unavoidable [9]. Hence, the

\footnotetext{
*This work is supported in part by funds provided by the US Department of Energy (DOE) under cooperative research agreement \#DF-FC02-94ER40818.
}

purpose of this work is to avoid the limitations of cooling by studying the low eigenfunctions of the Dirac operator directly in uncooled configurations.

One essential piece of background information concerns vacuum matrix elements of meson correlation functions $\left\langle\Omega\left|T J(x) J(0)^{\dagger}\right| \Omega\right\rangle$ in the vector and pseudoscalar channels[7]. Lattice calculations agree well with phenomenological dispersion analyses[2], and the strength in the vicinity of $1 \mathrm{fm}$ is strongly dominated by the rho and pion contributions.

\section{Eigenmodes of the Dirac operator}

Using the k-step Arnoldi method 10 we have calculated eigenmodes of the Wilson Dirac operator

$$
\begin{aligned}
D \psi_{x}= & \psi_{x}-\kappa \sum_{\mu}\left[\left(r-\gamma_{\mu}\right) u_{x, \mu} \psi_{x+\mu}\right. \\
& \left.+\left(r+\gamma_{\mu}\right) u_{x-\mu, \mu}^{\dagger} \psi_{x-\mu}\right]
\end{aligned}
$$

in several simple background configurations to understand their structure, as well as on $16^{4}$ lattices for quenched QCD at $\beta=5.85$ and for unquenched QCD at $\beta=5.5$ and $\kappa_{\text {sea }}=0.16$.

As is well known, in the free case, a discrete set of points starting at the lowest Matsubara mode $\left(0,0,0, \pm \pi / L_{t}\right)$ approximates the contin- 


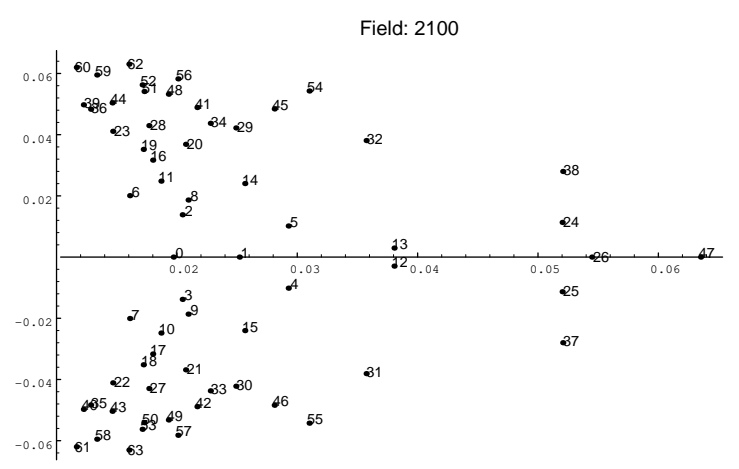

Figure 1. Lowest 64 eigenvalues of the Dirac operator for an unquenched gluon configuration.

uum spectrum $\frac{1}{m}[m+i|\vec{p}|]$ in the physical regime and unphysical fermion modes are pushed to large (real) masses. In the presence of an instanton of size $\rho$ at $x=0$, there are two lattice artifacts. Because of the Wilson term, the eigenvalue acquires a shift of approximately $\operatorname{Re} \lambda_{0}=\left\langle\psi_{0}|D| \psi_{0}\right\rangle=$ $3 \kappa a / \rho^{2}$. Also, in addition to a mode with zero imaginary part that approaches the continuum result

$$
\psi_{0}(x)_{s, \alpha}=u_{s, \alpha} \frac{\sqrt{2}}{\pi} \frac{\rho}{\left(x^{2}+\rho^{2}\right)^{3 / 2}}
$$

one spin and color component of the lowest Matsubara mode $\left(0,0,0, \pm \pi / L_{t}\right)$ mixes with the zero mode with a coefficient that goes to zero in the large volume limit. The eigenvalues for an instanton-anti-instanton pair are approximately

$$
\lambda=\frac{\lambda_{I}+\lambda_{A}}{2} \pm \sqrt{\frac{\left(\lambda_{I}-\lambda_{A}\right)^{2}}{4}-\left(T_{I A}\right)^{2}}
$$

so that when the interaction $T_{I A}$ exceeds the difference in the Wilson term shifts, complex conjugate pairs of eigenvalues move slightly off the real axis.

Fig. 11 shows the lowest 64 complex eigenvalues of the Dirac operator on an unquenched gluon configuration. Note that the eigenvalues of this uncooled configuration have just the structure expected from the pure one- and two-instanton studies above: isolated zeros along the real axis corresponding to individual instantons and complex conjugate pairs corresponding to instantonanti-instanton pairs. To set the scale, note that

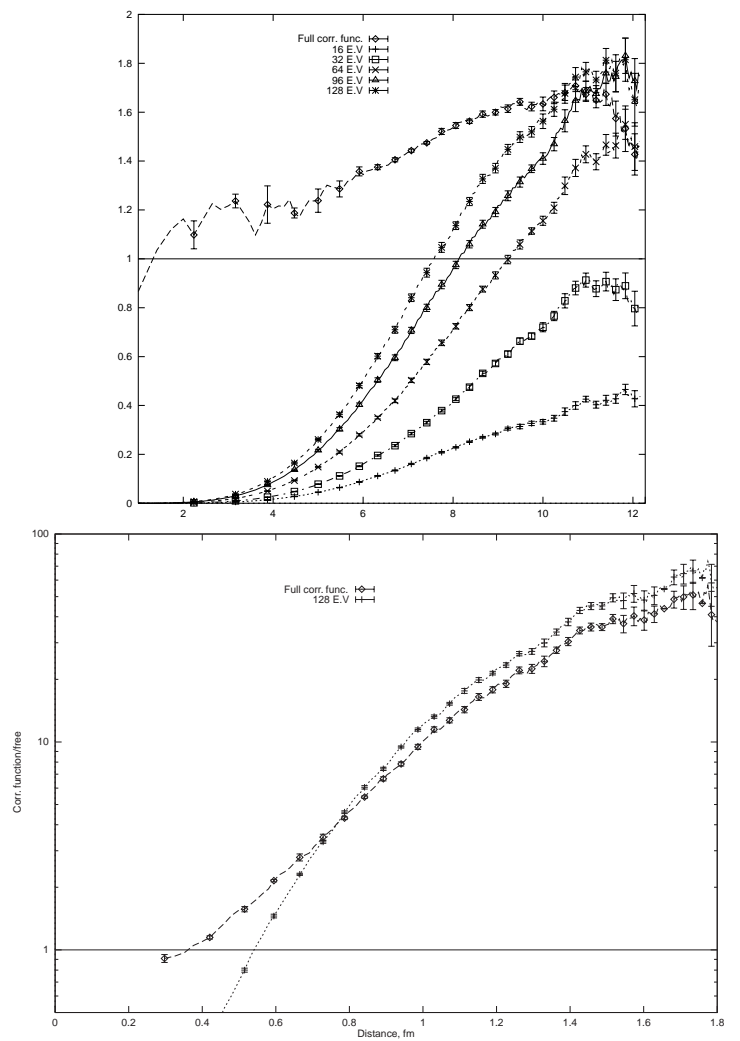

Figure 2. Contributions of low Dirac eigenmodes to the vector (upper graph) and pseudoscalar (lower graph) vacuum correlation functions.

the lowest Matsubara mode would occur at 0.06 on the imaginary axis, corresponding to $380 \mathrm{MeV}$, so all the conjugate pairs below this value are understood to be the results of zero modes.

\section{Zero mode expansion}

The Wilson-Dirac operator has the property that $D=\gamma_{5} D^{\dagger} \gamma_{5}$, which implies that $\left\langle\psi_{j}\left|\gamma_{5}\right| \psi_{i}\right\rangle=0$ unless $\lambda_{i}=\lambda_{j}^{*}$ and we may write the spectral representation of the propagator

$$
\left\langle x\left|D^{-1}\right| y\right\rangle=\sum_{i} \frac{\left\langle x \mid \psi_{i}\right\rangle\left\langle\psi_{\bar{i}}\left|\gamma_{5}\right| y\right\rangle}{\left\langle\psi_{\bar{i}}\left|\gamma_{5}\right| \psi_{i}\right\rangle \lambda_{i}}
$$

where $\lambda_{i}=\lambda_{\bar{i}}^{*}$. A clear indication of the role of zero modes in light hadron observables is the degree to which truncation of the expansion to 
the zero mode zone reproduces the result with the complete propagator.

Fig. 2 shows the result of truncating the vacuum correlation functions for the vector and pseudoscalar channels to include only low eigenmodes 11. On a $16^{4}$ lattice, the full propagator contains 786,432 modes. The top plot of Fig. 2 shows the result of including the lowest 16, 32, 64, 96, and finally 128 modes for an unquenched configuration with a $63 \mathrm{MeV}$ valence quark mass. Note that the first 64 modes reproduce most of the strength at $1 \mathrm{fm}$ (10 lattice spacings) where the correlator is saturated by the rho resonance peak, and by the time we include the first 128 modes, all the strength is accounted for. Similarly, the lower plot for a quenched configuration with a $23 \mathrm{MeV}$ quark mass shows that the lowest 128 modes also account for the analogous pion contribution to the pseudoscalar vacuum correlation function. Thus, without having to resort to cooling, by looking directly at the contribution of the lowest eigenfunctions, we have shown that the zero modes associated with instantons dominate the propagation of rho and pi mesons in the QCD vacuum.

\subsection{Localization}

Finally, it is interesting to ask whether the lattice zero mode eigenfunctions are localized on instantons. This was studied by plotting the quark density distribution for individual eigenmodes in the $x-z$ plane for all values of $y$ and $t$, and comparing with analogous plots of the action density. As expected, for a cooled configuration the eigenmodes correspond to linear combinations of localized zero modes at each of the instantons. What is truly remarkable, however, is that the eigenfunctions of the uncooled configurations also exhibit localized peaks at locations at which instantons are identified by cooling. Thus, in spite of the fluctuations several orders of magnitude larger than the instanton fields themselves, the light quarks essentially average out these fluctuations and produce localized peaks at the topological excitations. When one analyzes a number of eigenfunctions, one finds that all the instantons remaining after cooling correspond to localized quark fermion peaks in some eigenfunctions.
However, some fermion peaks are present for the initial gluon configurations that do not coincide with instantons that survive cooling, corresponding to instanton-anti-instanton pairs that were annihilated during cooling.

\section{Acknowledgments}

It is a pleasure to acknowledge the donation by Sun Microsystems of the 24 Gflops E5000 SMP cluster on which the most recent calculations were performed and the computer resources provided by NERSC with which this work was begun.

\section{REFERENCES}

1. M.-C. Chu, J.M. Grandy, S. Huang, and J.W. Negele, Phys. Rev. D49, 6039 (1994).

2. E.V. Shuryak, Rev. Mod. Phys. 65, 1 (1993), Nucl. Phys. B (Proc. Suppl.) 34, 107 (1994), and T. Schäffer and E.V. Shuryak, hepph/9610451 v2.

3. D.I. Dyakanov and V. Yu Petrov, Nucl. Phys. B245, 259 (1984); B272, 457 (1986).

4. G. 't Hooft, Phys. Rev. 14D, 3432 (1976).

5. E.V. Shuryak and J.J.M. Verbaarschot, Nucl. Phys. B410, 55 (1993); T. Schäffer, E.V. Shuryak, and J.J.M. Verbaarschot, Nucl. Phys. B412, 143 (1994).

6. B. Berg, Phys. Lett. 104B, 475 (1981); M. Teper, Nucl. Phys. B (Proc. Suppl.) 20, 159 (1991).

7. M.-C. Chu, J.M. Grandy, S. Huang, and J.W. Negele, Phys. Rev. Lett. 70, 225 (1993); Phys. Rev. D48, 3340 (1993).

8. P. de Forcrand, M. Garcia Pérez, and I.-O. Stamatescu, hep-lat/9701012 (1997).

9. R.C. Brower, T.L. Ivanenko, J.W. Negele, and K.N. Orginos, Nucl. Phys. B (Proc. Suppl.) 53, 547 (1997).

10. D. Sorensen, SIAM J. Matrix Anal. Appl. 13, 357 (1992).

11. T. Ivanenko, MIT Ph.D. dissertation (1997). 